\title{
Transaction
}

\section{Polyamide 6 Fibers with Superior Mechanical Properties : TPU Coating Techniques}

\author{
Baiju John ${ }^{* 1}$, Suguru Motokucho ${ }^{* 2}$, Ken Kojio ${ }^{* 2}$, and Mutsuhisa Furukawa ${ }^{* 1}$ \\ ${ }^{* 1}$ Division of Materials Science, Graduate School of Science and Technology, \\ ${ }^{* 2}$ Department of Materials Science and Engineering, Faculty of Engineering, \\ Nagasaki University, 1-14 Bunkyo-machi, Nagasaki 852-8521, Japan
}

\begin{abstract}
Thermoplastic polyurethane (TPU) was thin film coated on polyamide 6 (PA6) fibers by the reactive bulk and solution coating techniques. The thickness of the coating was $40 \mu \mathrm{m}$ for the reactive bulk coating technique and $20 \mu \mathrm{m}$ for the solution coating. The increase in crystallinity, the formation of the interface interaction region and the entry of TPU thin film coating into the micro-cracks under tensile stress resulted in the increase of strength as well as elongation of the TPU coated PA6 fibers. The microphase separated-structure and the orientation of the hard segment domains of the TPU thin film coating on the PA6 fiber surface had an impact on the force alignment of the PA6 fiber when under a mechanical stress. The hard segment domains of the TPU thin film which was prepared by a solution coating technique showed better alignment to the deformation force direction compared to the reactive bulk based one. This led to better mechanical strength of the former based TPU coated PA6 fiber. The TPU is a soft material and the improvement of mechanical property with same should be specially noted. So an understanding of both type of coating techniques is very useful in various industrial applications of PA6 fibers.
\end{abstract}

(Received 9 March, 2009 ; Accepted 9 May, 2009)

\section{Introduction}

Polyamide 6 (PA6) is a thermoplastic polymer widely used in our daily life such as textiles, engineering applications, biomedical and marine applications. The properties that account for the popularity of PA6 are the semi-crystalline nature, melting point, mechanical strength, good resistance to hydrocarbons, and the ease of fabrication and processing [1-4]. However, PA6 fibers are prone to break under mechanical stress due to the formation of micro cracks over the surface. Also, it undergoes weathering, UV degradation and abrasion. Thermoplastic polyurethanes (TPUs) have resistance towards abrasion and weathering; is composed of a soft segment formed by a polymer glycol and a hard segment formed by the diisocyanate and a chain extender. The soft segment can be classified as ether or ester series, including carbonate ones. Polycarbonate-based TPU have excellent resistance to heat, hydrolysis and resistance to ultraviolet radiation in comparison with ether series $[5,6]$. In the previous study, it was revealed that TPU thin film coating prepared by a reactive bulk coating technique, improved the performance of the PA6 fibers especially in the mechanical and weathering resistance aspects [7]. In the present study TPU thin film coated PA6 fibers were prepared by the reactive and solution based coating techniques and the impact of both the coating methods on the mechanical properties of the same were analyzed.

\section{Experimental}

\subsection{Materials}

Figure 1 shows the chemical structure of the raw materials used. Poly (hexamethylene carbonate) glycol (PCG) of an average molecular weight $\left(M_{\mathrm{n}}\right)$ of 1076 $\mathrm{g} / \mathrm{mol}$ and 4,4'-diphenylmethane diisocyanate (MDI) were supplied by AsahiKasei Chemicals Co., Ltd., (Tokyo, Japan) and Nippon Polyurethane Industry, Co., Ltd., (Tokyo, Japan), respectively. 1, 4-Butanediol (BD), used as the chain extender and N,N-Dimethylformamide (DMF) was purchased from Wako Chemical Co., Ltd., (Osaka, Japan). PCG was dried by passing the nitrogen gas under a reduced pressure at $100{ }^{\circ} \mathrm{C}$. To remove water, $\mathrm{BD}$ was distilled prior to its use. The PA6 monofilament $(\phi=520 \mu \mathrm{m})$ was purchased from Toray Co., Ltd, (Tokyo, Japan).

\subsection{Reactive bulk and solution coating methods}

The PA6 monofilament fibers were cleaned using distilled water in an ultrasonic water bath for $15 \mathrm{~min}$ and dried at room temperature. Polycarbonate glycol (PCG) 


$$
\begin{aligned}
& \text { Poly (hexamethylene carbonate) glycol } \\
& \text { (PCG) }
\end{aligned}
$$

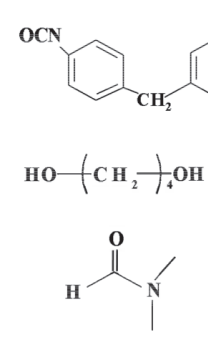

Fig. 1 Raw materials used for the preparation of TPU coating.

was bubbled with a nitrogen gas at $100{ }^{\circ} \mathrm{C}$ to remove moisture for $5 \mathrm{~h}$. TPU was prepared at an [NCO] / $[\mathrm{OH}]_{\mathrm{PCG}}$ ratio $(\mathrm{K})$ of 2.05 to 3.05 and an NCO INDEX $\left([\mathrm{NCO}] /[\mathrm{OH}]_{\mathrm{PCG}+\mathrm{BD}}\right)$ of 1.05 . PCG was mixed with $\mathrm{BD}$ at $100{ }^{\circ} \mathrm{C}$ and agitated for 3 min under a nitrogen atmosphere. MDI was added and agitated for $30 \mathrm{~s}$ and the whole mixture was vacuum agitated for $1 \mathrm{~min}$. The TPU adduct thus prepared was ready for the reactive bulk and solution based coating techniques.

In the case of reactive bulk coating method, the PA6 fiber was directly dipped into the TPU adduct prepared and the coated PA6 fiber was cured at $100{ }^{\circ} \mathrm{C}$ for $24 \mathrm{~h}$ in a moisture free chamber. In the solution coating method the prepared TPU adduct was made into a sheet in a centrifugal curing unit at $100{ }^{\circ} \mathrm{C}$ for $24 \mathrm{~h}$. The TPU sheet was then dissolved in DMF and a 20wt\% TPU solution was prepared. The PA6 fiber was dipped into the TPU solution medium and the coated fiber was dried in a moisture free chamber at $100{ }^{\circ} \mathrm{C}$ for $24 \mathrm{~h}$. The prepared fibers by both the methods were taken for analysis by various characterization methods. The sample was designated as PA6, PCG2-PA6 (R) and PCG3-PA6. They represents polyamide 6 fiber, polycarbonate based TPU thin film coated PA6 fiber for $\mathrm{K}=2$ and 3, respectively. ' $R$ ' and ' $S$ ' in the sample name represent TPU prepared by the reactive bulk and solution based coating techniques, respectively.

\subsection{Characterization}

The molecular arrangement and morphology were evaluated by polarized optical microscope (POM), field emission scanning electron microscopy (FE-SEM), differential scanning calorimetry (DSC), and a tensile tester.

POM images were obtained with a Nikon OPTIPHTO2-POL polarized optical microscope equipped with a Linkham TH500PM heating stage (Linkham scientific Instruments, UK). Field emission SEM (FESEM) images were taken with a JEOL JSM-7500 scanning electron microscope (Tokyo, Japan) operated at $5 \mathrm{kV}$. The samples were gold sputtered prior to SEM examination.

DSC was performed to determine the thermal behavior of PA6 fibers and TPU coated PA6 fibers. DSC curves were obtained with a Rigaku DSC 8230 differential scanning calorimeter (Rigaku Co. Ltd., Tokyo, Japan) from -150 to $250{ }^{\circ} \mathrm{C}$ at a heating rate of $10{ }^{\circ} \mathrm{C}$ $\min ^{-1}$ under a nitrogen atmosphere. The samples were cooled to approximately $-150{ }^{\circ} \mathrm{C}$ at a cooling rate of 6 $8{ }^{\circ} \mathrm{C} \min ^{-1}$, before the measurements.

Tensile tests were performed with an Instron type tensile tester (Tensilon, U-440 model, Orientec Co., Ltd., Tokyo Japan). The initial length and elongation rate were set at $30 \mathrm{~mm}$ and $10 \mathrm{~mm} \mathrm{~min}^{-1}$, respectively.

\section{Results and discussion}

\subsection{Morphological features}

Table 1 shows the properties of the PA6 fibers studied. The average thickness of the TPU coating on the PA6 fibers by the reactive bulk coating method was $40 \mu \mathrm{m}$ and it was $20 \mu \mathrm{m}$ for the solution method. In the case of the reactive bulk method, controlling the thickness of the TPU film was difficult due to the fast curing nature while in the case of solution method it was comparatively easy. The variation in thickness for both the methods was $\pm 5 \mu \mathrm{m}$. There was no variation in the density of TPU coated PA6 fibers for both the methods. Transparency of the TPU coating varied with the $\mathrm{K}$ value $([\mathrm{NCO}] /[\mathrm{OH}]$ ratio). The TPU coating of PCG2-PA6(R) fibers was more transparent than that of PCG3-PA6 (R). The same phenomenon was observed for the solution based ones also. The hard segment content of PCG3-PU was more than PCG2-PU due to the variation in the $\mathrm{K}$ values. Figure 2 (a) and (b) show the POM images for cross section of the PCG2-PA6 (R) fiber and the PCG2-PA6 (S)

Table 1 The properties of the TPU thin film coating by the reactive bulk and solution based coating techniques.

\begin{tabular}{lccccc}
\hline Fiber & K & $\begin{array}{c}\text { Coating } \\
\text { thickness } \\
\text { value }\end{array}$ & Density & $\begin{array}{c}\text { Coating } \\
\text { weight } \\
\text { fraction }\end{array}$ & Trans.* \\
& & $(\mu \mathrm{m})$ & $\left(\mathrm{g} / \mathrm{cm}^{3}\right)$ & $(\%)$ & \\
\hline PA6 & -- & -- & 1.22 & -- & High \\
Ann-PA6 & -- & -- & 1.22 & -- & High \\
\hline PCG2-PA6(R) & 2.05 & 40 & 1.23 & 20.3 & High \\
PCG3-PA6(R) & 3.05 & 40 & 1.24 & 28.6 & Medium \\
PCG2-PA6(S) & 2.05 & 20 & 1.21 & 9.89 & High \\
PCG3-PA6(S) & 3.05 & 20 & 1.22 & 13.8 & Medium \\
\hline PCG2-PU & 2.05 & -- & 1.13 & -- & High \\
PCG3-PU & 3.05 & -- & 1.16 & -- & Medium \\
\hline & * represents transparency/ appearance &
\end{tabular}



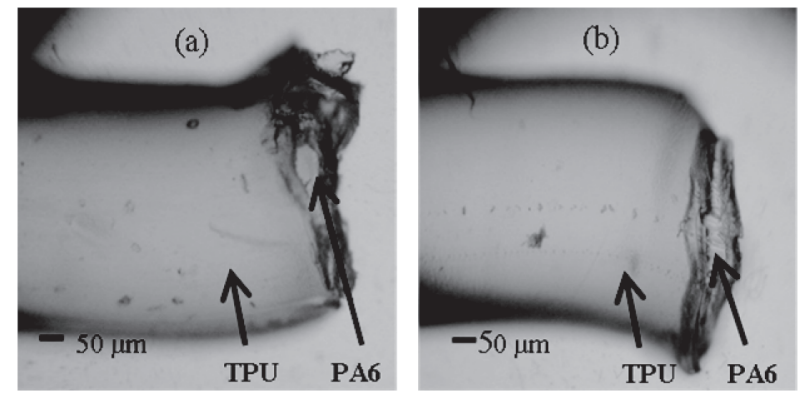

Fig. 2 (a), (b) Surface view of PCG2-PA6(R) and PCG2-PA6(S) fibers.

one. The images clearly suggest that TPU coating showed good compatibility with the PA6 fiber and had no cracks or other irregularities over it. An interface interaction region was observed at the interface of the fiber and the coating as shown in Figure $5(\mathrm{a}, \mathrm{c})$ for both the type of coating methods. The interface interaction comprised of equal parts from PA6 and TPU sides at the interface ; the width of the region was roughly $10 \mu \mathrm{m}$ for both the coating techniques.

\subsection{Thermal properties}

Figure 3 shows the DSC thermograms of the fibers studied. Table 2 shows the thermal properties of TPU coated PA6 fibers by the reactive bulk and solution based coating techniques. The glass transition temperature $\left(T_{\mathrm{g}}\right)$ of PA6 fiber was around $55{ }^{\circ} \mathrm{C}$. The $T_{\mathrm{g}}$ of soft segments of PCG2-PU and PCG3-PU was -5.8 and $-7.5{ }^{\circ} \mathrm{C}$. The melting temperatures of the hard segment domains of PCG2-PU and PCG3-PU were 158.9 and $160.5{ }^{\circ} \mathrm{C}$, respectively. Thus, TPU used for the study had a phase separation between the hard and soft segments. The arrangement of the microphase-separated structure over the PA6 fiber surface by the two coating techniques have

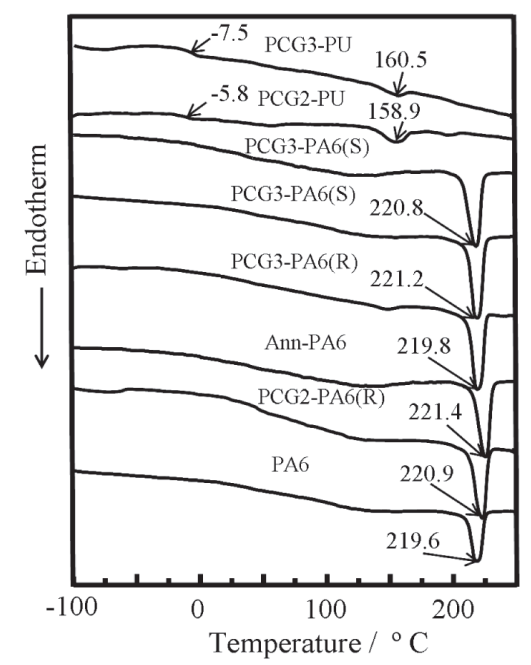

Fig. 3 DSC thermograms of the TPUs and reactive bulk coated and solution coated PA6 fibers.
Table 2 Thermal properties of the TPU coated PA6 fibers.

\begin{tabular}{lccccc}
\hline Fiber & $\begin{array}{c}\mathrm{K} \\
\text { value }\end{array}$ & $\begin{array}{c}\mathrm{T}_{\mathrm{g}} \\
\left({ }^{\circ} \mathrm{C}\right)\end{array}$ & $\begin{array}{c}\mathrm{T}_{\mathrm{m}} \\
\left({ }^{\circ} \mathrm{C}\right)\end{array}$ & $\begin{array}{c}\Delta \mathrm{H}_{\mathrm{f}} \\
(\mathrm{J} / \mathrm{g})\end{array}$ & $\begin{array}{c}\chi \\
(\%)\end{array}$ \\
\hline PA6 & -- & 55.1 & 219.6 & 48.6 & 21.1 \\
Ann-PA6 & -- & 51.2 & 221.4 & 58.4 & 25.3 \\
\hline PCG2-PA6(R) & 2.05 & 54.2 & 220.9 & 64.4 & 28.0 \\
PCG3-PA6(R) & 3.05 & 53.9 & 219.8 & 66.5 & 29.0 \\
PCG2-PA6(S) & 2.05 & 54.4 & 221.2 & 65.4 & 29.1 \\
PCG3-PA6(S) & 3.05 & 53.1 & 220.8 & 67.0 & 29.8 \\
\hline PCG2-PU & 2.05 & -5.8 & 158.9 & 1.4 & -- \\
PCG3-PU & 3.05 & -7.5 & 160.5 & 2.4 & -- \\
\end{tabular}

significant impact on the mechanical properties of the same, shall be discussed in the later section. The $T_{\mathrm{m}}$ of the PA6 fiber showed no variation with the TPU coating. Thus, the TPU thin film coatings have no significant effect on the $T_{\mathrm{m}}$ of PA6 fibers. The crystallinity of TPU coated PA6 fiber was calculated from the equation.

$$
\chi=\left(\Delta H_{\mathrm{f}} / \Delta H_{100}\right) \times 100 \%
$$

where, $\Delta H_{\mathrm{f}}$ represents the enthalpy of melting of the fiber and $\Delta H_{100}$ represents the enthalpy of melting of a $100 \%$ pure crystalline PA6 sample i.e. $230 \mathrm{~J} / \mathrm{g}[8,9]$. The TPU coated PA6 fibers prepared by both the coating techniques showed an increase in the crystallinity. This indicates that structural changes occurred in the PA6 fibers which led to the rearrangement in the crystalline regions [7]. The TPU coating on PA6 fiber by both the coating techniques is thermally stable. The increase of crystallinity definitely had an impact on the mechanical properties of PA6 fibers.

\subsection{Mechanical properties}

Figure 4 shows the stress-strain curves of PCG2-PA6 fibers by the reactive bulk and solution coating techniques. Table 3 shows the mechanical properties of TPU coated PA6 fibers by the reactive bulk and solution coating techniques. The strength and the elongation at break of TPU coated PA6 fibers increased, irrespective of the coating techniques. Besides, the Young's modulus also showed an increase. In the rapid process of fiber manufacture, rapid spinning and cooling takes place before the completion of growth of PA6 lamellar structures. This results in disordered regions all along the PA6 fibers. So, during annealing there will be a rearrangement in lamellar structures that result in the elongation of Ann-PA6 fibers [10]. But in this study, the TPU coated PA6 fibers showed a much better 


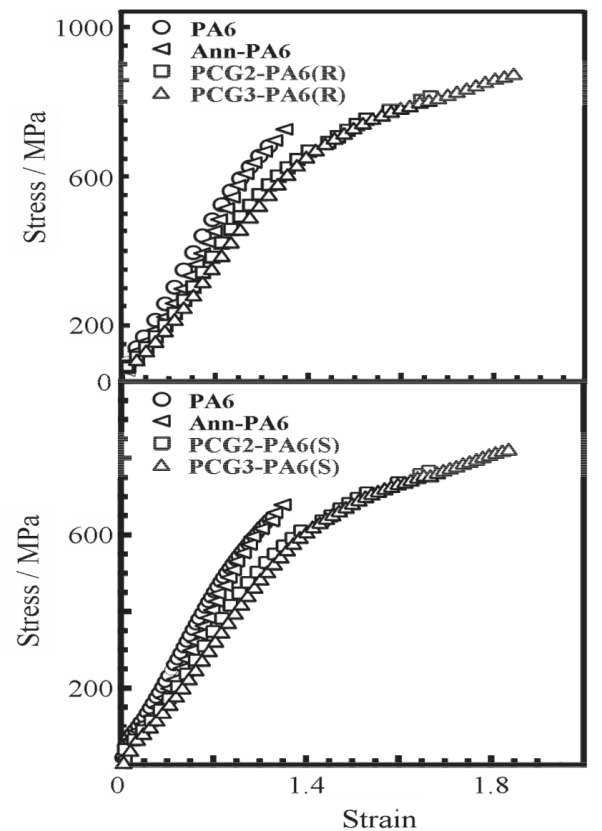

Fig. 4 (a), (b) Stress-strain curves of reactive bulk and solution coated PA6 fibers.

performance compared to the annealed ones. In the TPU coated PA6 fiber system, the regions that influence the mechanical strength are the inner fiber core, the interface interaction region and the outer TPU coating region. The strength of the interface interaction region is much higher than that of the outer layer TPU coating, which comprises of equal parts of PA6 and TPU coating [7]. Figure 5 shows interface interaction region of TPU coated PA6 fibers before and after subjecting a mechanical load equivalent to $90 \%$ of the break load. It can be seen that the TPU coating still adheres to the PA6 fiber even after the application of mechanical stress. This confirms the interaction through hydrogen bonding at the interface of PA6 and TPU. When PA6 fibers undergo tensile stress, micro-cracks are formed on the surface of fibers. The micro-cracks are clearly visible and the TPU coating has

Table 3 Mechanical properties of the TPU coated PA6 fibers

\begin{tabular}{lcccc}
\hline Fiber & $\begin{array}{c}\mathrm{K} \\
\text { value }\end{array}$ & $\begin{array}{c}\text { Tensile } \\
\text { strength } \\
(\mathrm{MPa})\end{array}$ & $\begin{array}{c}\text { Elongation } \\
\text { at break }\end{array}$ & $\begin{array}{c}\text { Young's } \\
\text { Modulus } \\
(\mathrm{GPa})\end{array}$ \\
\hline PA6 & & 650.5 & 1.3 & 2.85 \\
Ann-PA6 & 681.2 & 1.4 & 3.22 \\
\hline PCG2-PA6(R) & 2.05 & 769.6 & 1.7 & 3.55 \\
PCG3-PA6(R) & 3.05 & 810.9 & 1.9 & 3.82 \\
PCG2-PA6(S) & 2.05 & 863.5 & 1.7 & 3.56 \\
PCG3-PA6(S) & 3.05 & 840.3 & 1.7 & 3.87 \\
\hline PCG2-PU & 2.05 & 26.6 & 1.0 & $\begin{array}{c}26.1 \\
(\mathrm{MPa})\end{array}$ \\
PCG3-PU & 3.05 & 28.6 & 1.6 & $\begin{array}{c}28.2 \\
(\mathrm{MPa})\end{array}$ \\
\hline
\end{tabular}

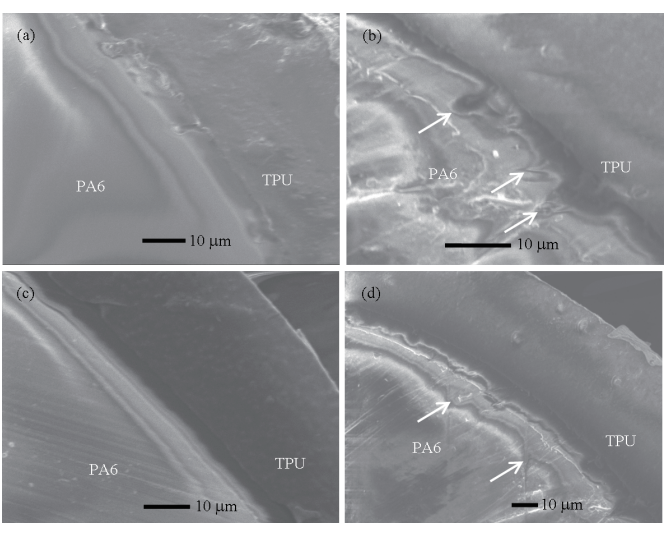

Fig. 5 (a), (b) FE-SEM images of the interface interaction region of PCG2-PA6(R) before and after the application of tensile load. (c), (d), represents the interface interaction region of PCG2-PA6(S) before and after the application of tensile load. The micro-cracks are clearly visible.

entered into the cracks during the deformation of the fiber. During the application of tensile load, the interface interaction region got stiffened leading to the increase of width of the same. The entry of TPU coating into the micro-cracks and the interface stiffening led to the increase of strength and elongation of TPU coated PA6 fibers. The thickness of solution based coating technique was less compared to the reactive bulk based one. But the strength of former based coated PA6 fibers was more than the latter one. PA6 and thermoplastic polyurethanes (TPU) are both polarizing polymers and so when both interact the possibility of structural rearrangements cannot be excluded. In the case of PA6 fibers the arrangement of lamellar domains is an important factor regarding the fiber mechanical performance. Since the PA6 fiber used was the same for the both the techniques, there was no lamellar rearrangement with the type of coating methods. So the impact of the microphase separated structure of the TPU coating has to be closely looked upon. Even though the microphase separation of TPU used for the both the thin film coating techniques was the same, the alignment of the hard segment domain on the fiber surface played a significant role on the mechanical property. Figure 6 shows the arrangement of microphase structure based on the reactive bulk and solution based coating techniques on PA6 fiber. The hard segment domain of the solution based ones was aligned more parallel to the PA6 lamellar regions. The fast curing and the presence of active isocyanate species in the reactive bulk coating caused the irregular arrangement of the hard segment domains. In the case of solution coating there was no curing and active isocyanates species was absent, the alignment of hard segments is regular. So when the mechanical load was 

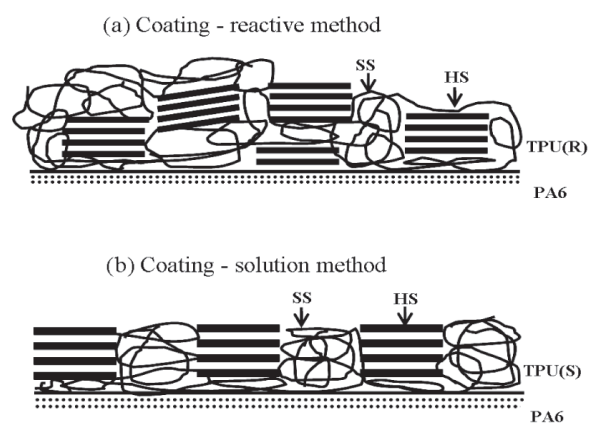

Fig. 6 The microphase structure of the TPU coating based on the reactive bulk and solution based coating techniques before the application of tensile load.

applied to the TPU coated PA6 fiber, the hard segment domains of the solution based coating got aligned to the applied deforming force direction as shown in Figure 7. In the case of the reactive bulk based ones, even though there was an alignment parallel to the applied force direction, the irregular arrangement of hard segment domains nullified the applied force balance towards the load direction. Thus the solution based coating showed more strength even though the thickness of the TPU coating was less when compared with the other.

\section{Conclusion}

The TPU thin film proved to be an excellent coating for the PA6 fibers. The coating did not sacrifice the appearance or the flexibility of the fiber and improved the physical properties significantly. Both the coating methods increased the strength as well as the elongation of the coated PA6 fibers. The improvement of the mechanical property of TPU coated fibers can be attributed to three factors; namely, the increase of crystallinity of the PA6 fiber with the coating, the role played by the interface interaction region, and the entry of TPU coating into the micro-cracks when a mechanical load is applied on the fiber. The mechanical strength of TPU coated PA6 fibers coated by solution coating technique was more than the reactive bulk based one. This was due to the better alignment of the hard segment domains of the former based TPU in parallel to the
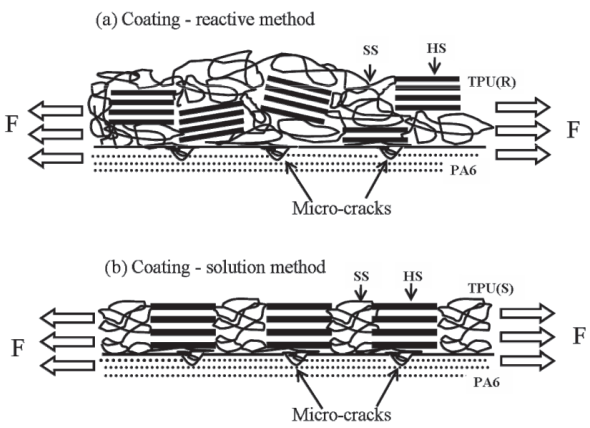

Fig. 7 The microphase structure of the TPU coating based on the reactive bulk and solution based coating techniques after the application of mechanical load. ' $F$ ' denotes the deformation force.

lamellar direction of PA6 fibers compared to the latter. The impact of hard segment alignment on the mechanical properties of PA6 fibers have not been reported yet. The two coating method are very convenient and the user can select the coating technique depending on the type of application of the PA6 fiber.

\section{References}

1. M. Palabiyik, and S. Bahadur, Wear, 246,149 (2000).

2. M. Xantos, Polymer Engineering and Science, 31, 929, (1991).

3. C. Ramesh, Macromolecules, 32, 5704 (1999).

4. N.S. Murthy, Macromolecules, 20, 309, (1987).

5. M. Furukawa, Y. Hamada, and K. Kojio, Journal of Polymer Science Part B : Polymer Physics, 41, 235, (2003).

6. K. Kojio, T. Fukumaru, and M. Furukawa, Macromolecules, 37, 3287, (2004).

7. B. John, K. Kojio and M. Furukawa, Polymer Journal, 41, 319, (2009).

8. J.T. Haponuik, Journal of Thermal Analysis, 60, 45, (2000).

9. J. Maillo, P. Pages, E.Vallejo, T. Lacorte, and J. Gacen, European Polymer Journal, 41, 753, (2005).

10. Polymer science, "Volume 1- A material science handbook", A.D. Jekins (Eds), North-Holland Co. London (1972). 